\title{
Phytosterol content and the campesterol: sitosterol ratio influence cotton fiber development: role of phytosterols in cell elongation
}

\author{
Shasha Deng ${ }^{\dagger}$, Ting Wei ${ }^{\dagger}$, Kunling Tan, Mingyu Hu, Fang Li, Yunlan Zhai, Shue Ye, \\ Yuehua Xiao, Lei Hou, Yan Pei \& Ming Luo* \\ Key Laboratory of Biotechnology and Crop Quality Improvement, Ministry of Agriculture/Biotechnology Research Center, Southwest \\ University, Chongqing 400716, China
}

Received May 14, 2015; accepted July 20, 2015; published online January 22, 2016

\begin{abstract}
Phytosterols play an important role in plant growth and development, including cell division, cell elongation, embryogenesis, cellulose biosynthesis, and cell wall formation. Cotton fiber, which undergoes synchronous cell elongation and a large amount of cellulose synthesis, is an ideal model for the study of plant cell elongation and cell wall biogenesis. The role of phytosterols in fiber growth was investigated by treating the fibers with tridemorph, a sterol biosynthetic inhibitor. The inhibition of phytosterol biosynthesis resulted in an apparent suppression of fiber elongation in vitro or in planta. The determination of phytosterol quantity indicated that sitosterol and campesterol were the major phytosterols in cotton fibers; moreover, higher concentrations of these phytosterols were observed during the period of rapid elongation of fibers. Furthermore, the decrease and increase in campesterol:sitosterol ratio was associated with the increase and decease in speed of elongation, respectively, during the elongation stage. The increase in the ratio was associated with the transition from cell elongation to secondary cell wall synthesis. In addition, a number of phytosterol biosynthetic genes were down-regulated in the short fibers of ligon lintless-1 mutant, compared to its near-isogenic wild-type TM-1. These results demonstrated that phytosterols play a crucial role in cotton fiber development, and particularly in fiber elongation.
\end{abstract}

cotton fiber, phytosterols, gene expression, tridemorph, ligon lintless-1

Citation: $\quad$ Deng, S., Wei, T., Tan, K., Hu, M., Li, F., Zhai, Y., Ye, S., Xiao, Y., Hou, L., Pei, Y., and Luo, M. (2016). Phytosterol content and the campesterol:sitosterol ratio influence cotton fiber development: role of phytosterols in cell elongation. Sci China Life Sci 59, 183-193. doi: $10.1007 / \mathrm{s} 11427-015-4992-3$

\section{INTRODUCTION}

Cotton (Gossypium hirsutum L.) is the leading fiber crop in the world. Cotton fibers are single elongated cells derived from the epidermal layer of the ovule. Fiber cell development includes four discrete but partially overlapping stages: initiation, elongation, secondary cell-wall accumulation, and maturation (Basra and Malik, 1984). Fiber initiation is visible on the epidermal surface of ovules on the day of anthesis, and is followed by cell elongation (Ryser, 2000). During

$\dagger$ Contributed equally to this work.

*Corresponding author (email: luomingyuan@swu.edu.cn) the elongation period, fiber cells elongate rapidly from three to five days post-anthesis (DPA), peak at around 10 DPA, and eventually grow up to 30 to $40 \mathrm{~mm}$ in length (Basra and Sukumar, 2000). Cotton fibers are among the longest cells characterized in the plant kingdom, and are not interrupted by cell division for a relatively long period; therefore, these fibers are used in the study of cell elongation and cell wall deposition (Cao, 2015; Deng et al., 2012; Lee et al., 2007; Li et al., 2015; Luo et al., 2007; Pei, 2015; Shan et al., 2014; Shi et al., 2006; Xiao et al., 2010; Yang et al., 2006; Yang et al., 2014; Zhang et al., 2011; Zhang et al., 2015).

Sterols are isoprenoid-derived molecules with essential functions in eukaryotes (Benveniste, 2004). Cholesterol is 
by far the most abundant sterol in vertebrates. Higher plants usually synthesize a complex mixture of various sterols, commonly referred to as phytosterols, among which sitosterol, campesterol, and stigmasterol are predominant forms (Benveniste, 2004). Campesterol serves as a precursor of brassinosteroid (BR) synthesis; influences the level of active BR; and regulates a number of physiological activities in plant development, such as cell elongation, stamen and pollen development, xylem differentiation, stress tolerance, and pathogen resistance (Asami et al., 2005; Choe et al., 2000; Chu et al., 2006; Fridman and Savaldi-Goldstein, 2013; Fujioka and Yokota, 2003; Gudesblat and Russinova, 2011; Williams, 2011). On the other hand, phytosterols are essential structural components that affect the biophysical properties of membranes, such as permeability and fluidity (Kim et al., 2005; Neelakandan et al., 2010; Schaller, 2003, 2004). In fact, recent studies have shown that phytosterol composition in the plasma membrane affects proper functioning of auxin transporters (Men et al., 2008; Souter et al., 2002, 2004; Willemsen et al., 2003). In addition, phytosterol derivatives are involved in many biological processes such as cell division, cell elongation, cell polarity, cellulose biosynthesis, cell wall formation, and embryonic pattern formation, by functioning as signaling molecules independent of BRs or by modulating the activity of membrane-bound enzymes (Boutté and Grebe, 2009; Carland et al., 2002; Clouse, 2000, 2002; Ovečka et al., 2010; Schaeffer et al., 2001; Schrick et al., 2000, 2002, 2004; Suzuki et al., 2009; Topping et al., 1997). These studies revealed the essential role of phytosterols in plant growth and development.

One of the most remarkable features of fiber development is rapid and synchronous elongation. A large number of phytosterols, important constituents of the cell membrane, must be synthesized to meet the needs of the dramatic increase in cell size during rapid elongation. Previous studies have shown that genes involved in phytosterol biosynthesis, such as GhSMT2-1 (sterol methyltransferase 2), GhSMT2-2, GhSMT1 (sterol methyltransferase 1), GhCYP$51 G 1$ (obtusifoliol 14 $\alpha$-demethylase), and GhHYDRAl $\left(\Delta^{8}-\Delta^{7}\right.$-sterol isomerase), are preferentially expressed in rapidly elongating fibers (Gou et al., 2007; Liu et al., 2012; Luo et al., 2008; Padmalatha et al., 2012; Shi et al., 2006; Tan et al., 2009; Wanjiea et al., 2005; Zang et al., 2011). Furthermore, BRs have been reported to play an important role in fiber growth (Luo et al., 2007; Kasukabe et al., 2001; Shi et al., 2006; Sun et al., 2004, 2005; Sun and Allen, 2005). However, the specific role of phytosterols in fiber development remains largely unknown.

In this study, we attempted to illuminate the role of phytosterols in the growth and development of cotton fiber cells by analyzing the contents of three major phytosterols: campesterol, sitosterol, and stigmasterol, in fiber cells, and comparing the expression of genes involved in phytosterol biosynthesis between ligon lintless-1 (li-l) mutant (a super short fiber mutant in upland cotton, which has a length of only 4-8 mm) and its near-isogenic wild-type TM-1 (Liu et al., 2012). We observed a plateau in phytosterol content and the campesterol to sitosterol ratio following fiber cell development; moreover, the results of our study indicated that a disturbance in phytosterol biosynthesis suppressed the fiber elongation process. These results indicated that phytosterols might play important roles in fiber development, specifically fiber elongation.

\section{RESULTS}

\section{Tridemorph suppresses the elongation of fiber cells}

Sterol biosynthesis inhibitors are effective tools for probing the regulatory functions of sterols across different kingdoms (Claude et al., 1996; He et al., 2003). To investigate the role of phytosterols in the development of cotton fiber cell, we used tridemorph, a sterol biosynthesis inhibitor, to mimic the effect of sterol deficiency (He et al., 2003). Tridemorph induced a significant inhibition in fiber elongation. In the ovule culture experiment, the length of fibers on ovules cultured on medium containing $0.625,1.25$, and $2.5 \mathrm{mg} \mathrm{L}^{-1}$ tridemorph were $(5.06 \pm 1.36),(2.32 \pm 0.73)$, and $(1.59 \pm 0.38)$ $\mathrm{mm}$, respectively, which were much shorter than that of the control $((10.78 \pm 1.61) \mathrm{mm}$, Figure 1A). In addition to the fiber length, tridemorph also inhibited ovule growth. The ovule size decreased with the increase in tridemorph concentration (Figure 1B). Planta experiments further confirmed the inhibitory effect of tridemorph on fiber elongation. The length of treated fibers was $(27.22 \pm 1.18) \mathrm{mm}$, which was reduced by $9.27 \%$ compared to the control $((30 \pm 1.64) \mathrm{mm}$, Figure $2 \mathrm{~A}$ and $\mathrm{B})$. As a result, we observed a decrease in the lint index and lint percentage of treated cotton (Figure 2C and D). These results indicated that the disruption of phytosterol biosynthesis suppressed in vitro and in vivo fiber elongation, suggesting the importance of phytosterols in fiber elongation.

\section{Sitosterol is the major phytosterol in developing fibers, and the ratio of campesterol to sitosterol may be associ- ated with the speed of elongation}

As shown above, phytosterols are crucial for normal growth of the cotton fiber. In order to elucidate the phytosterol profile in cotton fiber cells, we measured the contents of three phytosterols: sitosterol, campesterol, and stigmasterol, in the developing fibers of upland cotton variety Xuzhou 142 by gas chromatography-mass spectrometer (GC-MS). The gas chromatogram of three phytosterol standards, and the fiber cell and ovule extracts are shown in Figure 3. Under the conditions employed in our experiment, we achieved a baseline separation of the three components. The retention time of sitosterol, stigmasterol, and campesterol was approximately $16.881,15.651$, and $15.183 \mathrm{~min}$, respectively. Typical fragment ions, which provided the structural information of sterols, and the molecular ion peaks of three phy- 

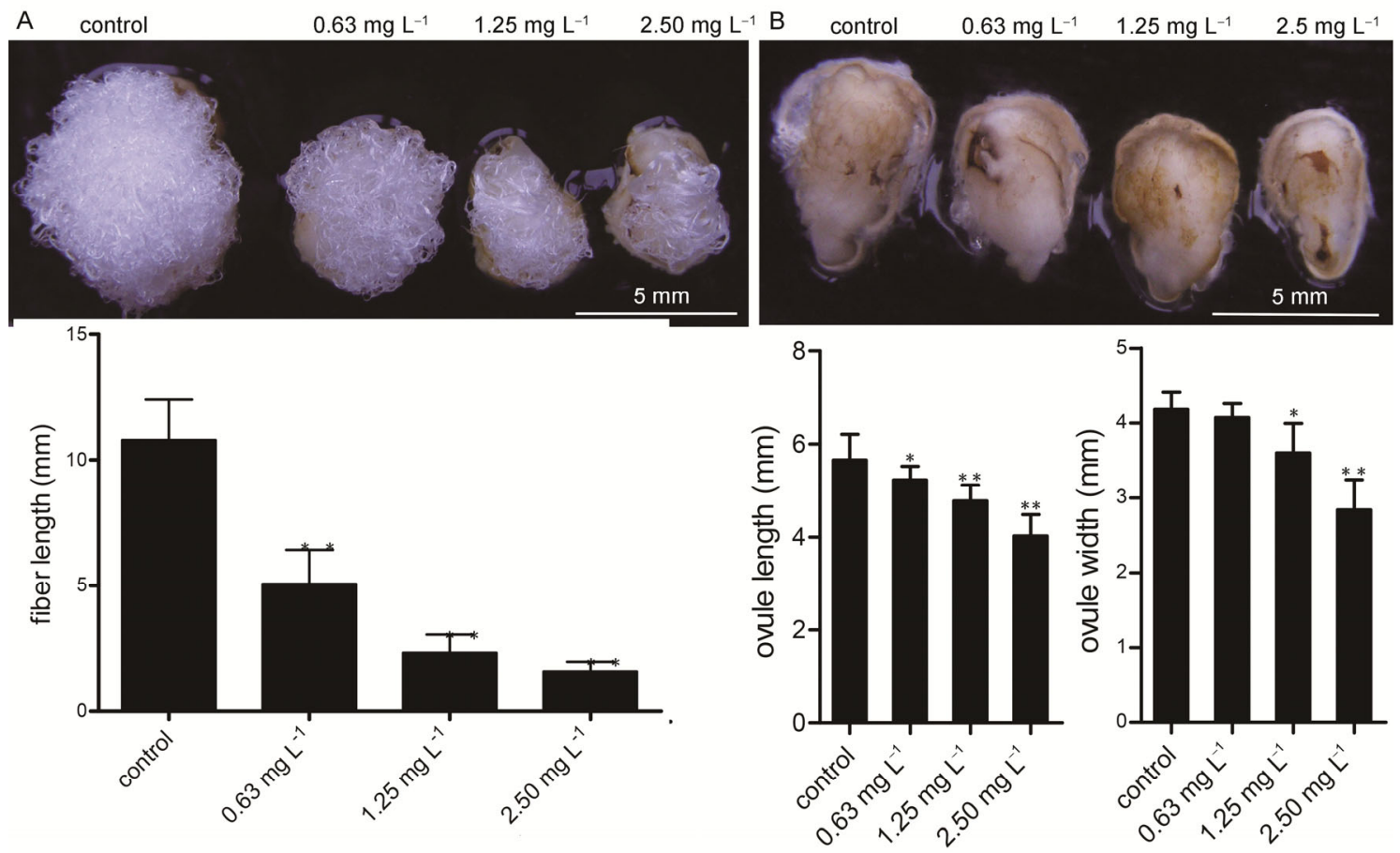

Figure 1 Inhibitory effect of tridemorph on fiber length and ovule growth in vitro. A, Effect of tridemorph on fiber growth. Ovules were grown in a floating culture on BT liquid medium without (control) and with tridemorph (concentration: 0.63, 1.25, and $2.5 \mathrm{mg} \mathrm{L}^{-1}$ ). Error bars represent the SD for five independent experiments. B, Effect of tridemorph on ovule growth. Ovule size was estimated based on its length (left) and width (right). Error bars represent the SD for five independent experiments. The data was evaluated by Student's $t$-test to determine statistical significance. *: $P<0.05$; **: $P<0.01$.

tosterols, were observed in the mass spectrogram; these were consistent with the peaks submitted to the standard library of mass spectrograms (Figure 4).

Overall, the level of sitosterol in the 6-30 DPA fibers was much higher than those of campesterol and stigmasterol. The average quantity of sitosterol was 4.7, 6.6, 4.3, and 7.6-fold greater than that of campesterol in 6-, 11-, 20-, and 30-DPA fibers, respectively. During the fiber developmental process, the sitosterol content increased rapidly from 6 DPA, peaked at 8 DPA, and decreased slightly from 8-20 DPA. The sitosterol content slightly increased from 20 to 30 DPA; however, the low level was maintained (Figure 5A). The change in campesterol content was moderate between 6 and 15 DPA and 20 and 30 DPA; however, an obvious decline was observed between 15 and 20 DPA. In contrast, the fibers contained very low levels of stigmasterol, which was nearly undetectable before 15 DPA. Stigmasterol was detectable in the 20-DPA and 25-DPA fibers, but at very low concentrations (Figure 5A). We observed a subsequent plateau in the concentration (Figure 5A). These results indicated that sitosterol is the major phytosterol in cotton fibers, especially during the stage of elongation (from 3 to 15 DPA), suggesting the important role of this phytosterol in cell elongation.

The ratio of campesterol to sitosterol was reported to play a key role in fitting the growth requirements and membrane integrity (Boutté and Grebe, 2009; Carland et al.,
2002; Clouse, 2000; Ovečka et al., 2010; Schaeffer et al., 2001; Suzuki et al., 2009). The ratio of campesterol to sitosterol decreased gradually during the early stage of fiber elongation (from 6 to $11 \mathrm{DPA}$ ), during which the speed of fiber elongation is accelerated. The ratio then increased rapidly from 11 to 20 DPA; this period included two stages: the late stage of rapid elongation and the transition stage of cell elongation to the onset of secondary cell wall synthesis. The speed of fiber elongation declined from peak to zero during this period. The ratio decreased drastically after cessation elongation ( 20 DPA) (Figure 5B). These results indicated that the ratio of campesterol to sitosterol was negatively correlated with the speed of fiber elongation. The ratio of campesterol to sitosterol was suggested to be a key factor influencing fiber elongation.

\section{Sitosterol and campesterol contents in elongating fibers of the short fiber mutant $l i-1$ were lower than those of TM-1}

The $l i-1$ mutant, characterized by abnormal lint fiber development, bears very short lint fibers similar to the fuzz fibers on seeds. Prior to 2 DPA, this mutant displayed a similar fiber initiation and elongation behavior as its isogenic wild-type line TM-1. However, after 2 DPA and during the rapid elongation stage, the mutant fibers cease to elongate and become fuzz-like fibers (Liu et al., 2012). Therefore, $l i-1$ has been used in the study of molecular 

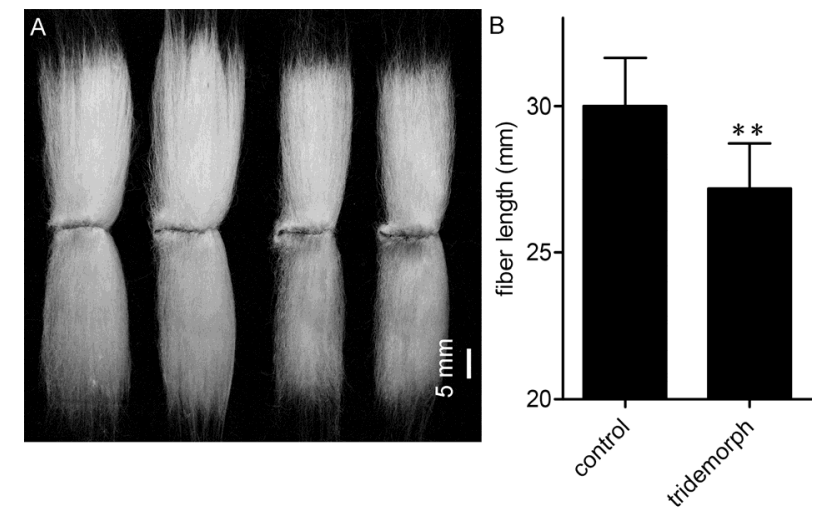

C
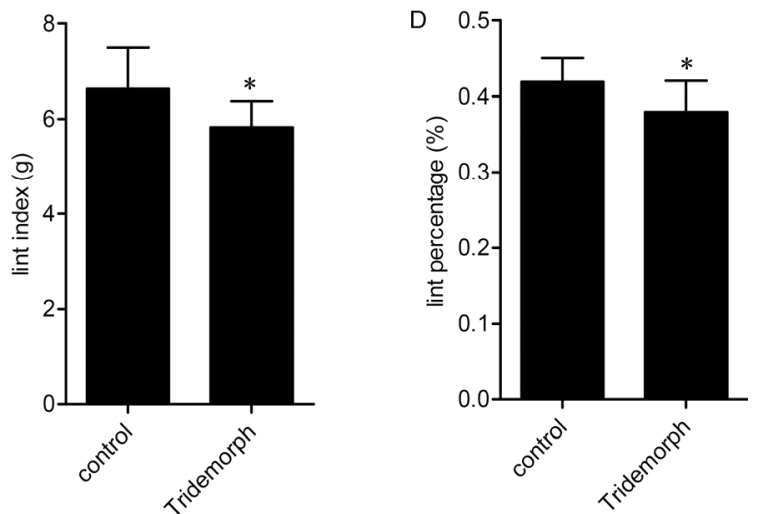

Figure 2 Tridemorph induced inhibition of fiber length in planta. A, Mature fibers on the seeds of tridemorph-treated $\left(25 \mathrm{mg} \mathrm{L}^{-1}\right.$ tridemorph, from 0 to $30 \mathrm{DPA}$, once every five days) and control cotton plants. B, Length of fibers harvested from the treated and control cotton plants. Error bars represent the SD for twenty seeds. C, Lint index of the control and tridemorph-treated cotton. Error bars represent the SD for three independent experiments. D, Lint percentage of the control and tridemorph-treated fiber. Error bars represent the SD for nine independent experiments. Lint index refers to the weight of fibers from 100 seeds ( $\mathrm{g}$ lint/100 seeds). Lint percentage refers to the lint (fiber) fraction of seed cotton (lint and seed). The data was evaluated by Student's $t$-test to determine statistical significance. *: $P<0.05$; **: $P<0.01$.

events involved in fiber cell elongation. To further elucidate the role of phytosterols in fiber elongation, we compared the contents of the three phytosterols in 10-DPA fibers of $l i-1$ mutant to those of TM-1. The sitosterol (major phytosterol) content was two-fold higher in TM-1 fibers $((0.385 \pm 0.044)$ $\left.\mathrm{mg} \mathrm{g}^{-1} \mathrm{DW}^{-1}\right)$ than that in $l i-1 \quad\left((0.192 \pm 0.068) \mathrm{mg} \mathrm{g}^{-1}\right.$ $\left.\mathrm{DW}^{-1}\right)$. The campesterol content was 3.3-fold higher in TM1 fibers $\left((0.076 \pm 0.008) \mathrm{mg} \mathrm{g}^{-1} \mathrm{DW}^{-1}\right)$ than that in the mutant $\left((0.023 \pm 0.008) \mathrm{mg} \mathrm{g}^{-1} \mathrm{DW}^{-1}\right)$. No stigmasterol was detected in the TM-1 fiber, while a trace was observed in the $l i-1$ fibers (Figure 6). These results indicated that the phytosterol biosynthesis was impaired in mutant fibers during elongation.

\section{Genes involved in phytosterol biosynthesis were down-regulated in the $l i-1$ mutant}

Genes involved in the phytosterol biosynthesis pathway have been characterized from Arabidopsis, and they include SMT1, SMT2, FK (FACKEL, sterol C-14 reductase), HYD1
(HYDRA1, $\Delta^{8}-\Delta^{7}$-sterol isomerase), DWF5 (DWARF5, sterol $\Delta^{7}$-reductase), and DWF7 (DWARF7, $\Delta^{7}$-sterol C-5 desaturase) (Benveniste, 2004). Their homologues in upland cotton (Gossypium hirsutum L.) have been cloned in recent years (Luo et al., 2007, 2008; Shi et al., 2006; Tan et al., 2009; Zang et al., 2011). We compared the level of expression of twelve genes associated with phytosterol biosynthesis between the fibers and ovules of the $l i-1$ mutant and TM-1. We observed no significant difference in the gene expression between $l i-1$ and TM-1 in the 0-DPA ovules (with fibers). Among the twelve genes investigated, the level of expression of seven genes (GhSMOI (sterol $4 \alpha$-methyl oxidase), GhCYP51G1, GhFK, GhSMT2-1, GhDWF7, GhDWF5, and GhDET2 (DE-ETIOLATED 2, steroid $5 \alpha$-reductase)) was significantly down-regulated in $l i-1$ than that in TM-1 in 6-DPA ovules (with fibers); particularly, we observed a 5-fold decrease in the transcript of GhDET2 in the mutant. The transcripts of eight genes were significantly lower in $l i-1$ than in TM-1 in the 10-DPA fibers (Figure 7). However, the level of expression of GhCYP710-1 and GhCYP710-2 increased in 6-DPA ovules (with fiber) and 10-DPA fibers of $l i-1$, respectively (Figure 7). CYP710 is involved in the conversion of sitosterol to stigmasterol (Benveniste, 2004). The increase in GhCYP710 expression in $l i-1$ was consistent with the presence of stigmasterol in the 10-DPA mutant fibers (Figure 6). These results indicated that phytosterol biosynthesis was weakened in mutant fibers at the elongation stage.

\section{DISCUSSION}

\section{Role of phytosterols in the elongation of cotton fibers}

Cotton fibers are highly elongated single cells. Phytosterols are components of the cell membrane; therefore, the phytosterol content must increase with the increase in membrane size during fiber cell elongation. We observed a high concentration of sitosterol, a major phytosterol in cotton fiber, which was consistent with the observations of previous reports (Gou et al., 2007; Wanjiea et al., 2005). The biosynthetic genes of phytosterols, such as GhSMT2-1, GhSMT2-2, GhSMT1, GhCYP51G1, and GhHYDRA1, are preferentially expressed in the rapidly elongating fibers (Gou et al., 2007; Liu et al., 2012; Luo et al., 2008; Padmalatha et al., 2012; Shi et al., 2006; Tan et al., 2009; Wanjiea et al., 2005; Zang et al., 2011), indicating that phytosterols was essential for fiber elongation. Inhibition of phytosterol biosynthesis by tridemorph suppressed fiber elongation in vivo or in planta. We observed a decrease in sitosterol and campesterol content in the fibers of the $l i-1$ mutant compared to the wild type TM-1. A number of phytosterol biosynthetic genes were correspondingly down-regulated in the $l i-1$ fiber, which was consistent with the results of a previous transcriptome analysis of early cotton fiber elongation in the $l i-l$ mutant (Liu et al., 2012). Low levels of sitosterol and campesterol were observed in 

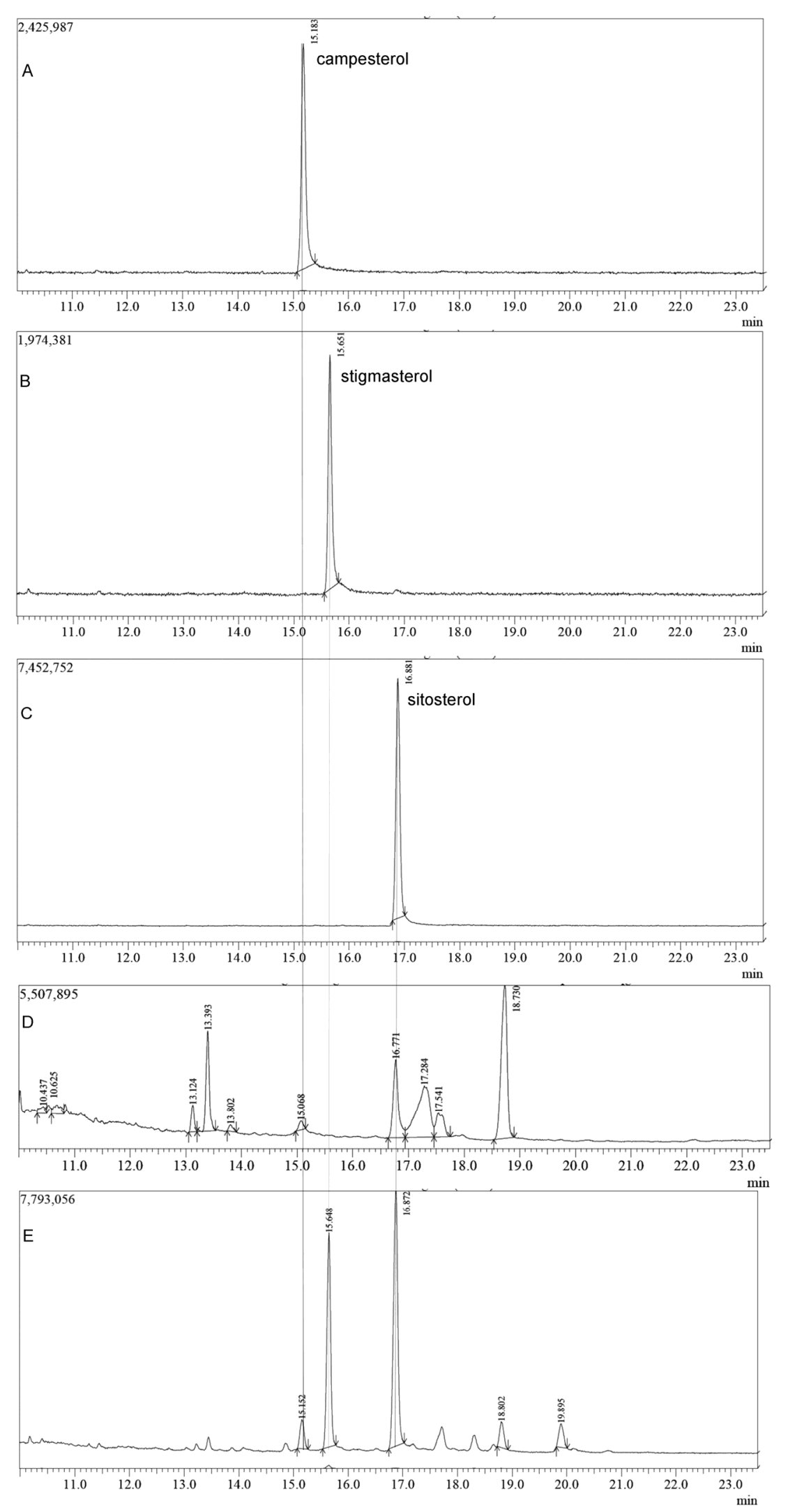

Figure 3 Gas chromatograms of three sterols (and their relevant peaks) in the extract of fiber cells and ovules. A, Gas chromatogram of sitosterol. B, Gas chromatogram of stigmasterol. C, Gas chromatogram of campesterol. D, Gas chromatogram of the 15-DPA fiber cell extract. Stigmasterol was not detected in this extract. E, gas chromatogram of the 10-DPA ovule extract. Stigmasterol was detected in the 10-DPA ovules. The relevant peaks were clearly separated in the fiber and ovule extract. The analytical conditions are detailed in the Methods section. 

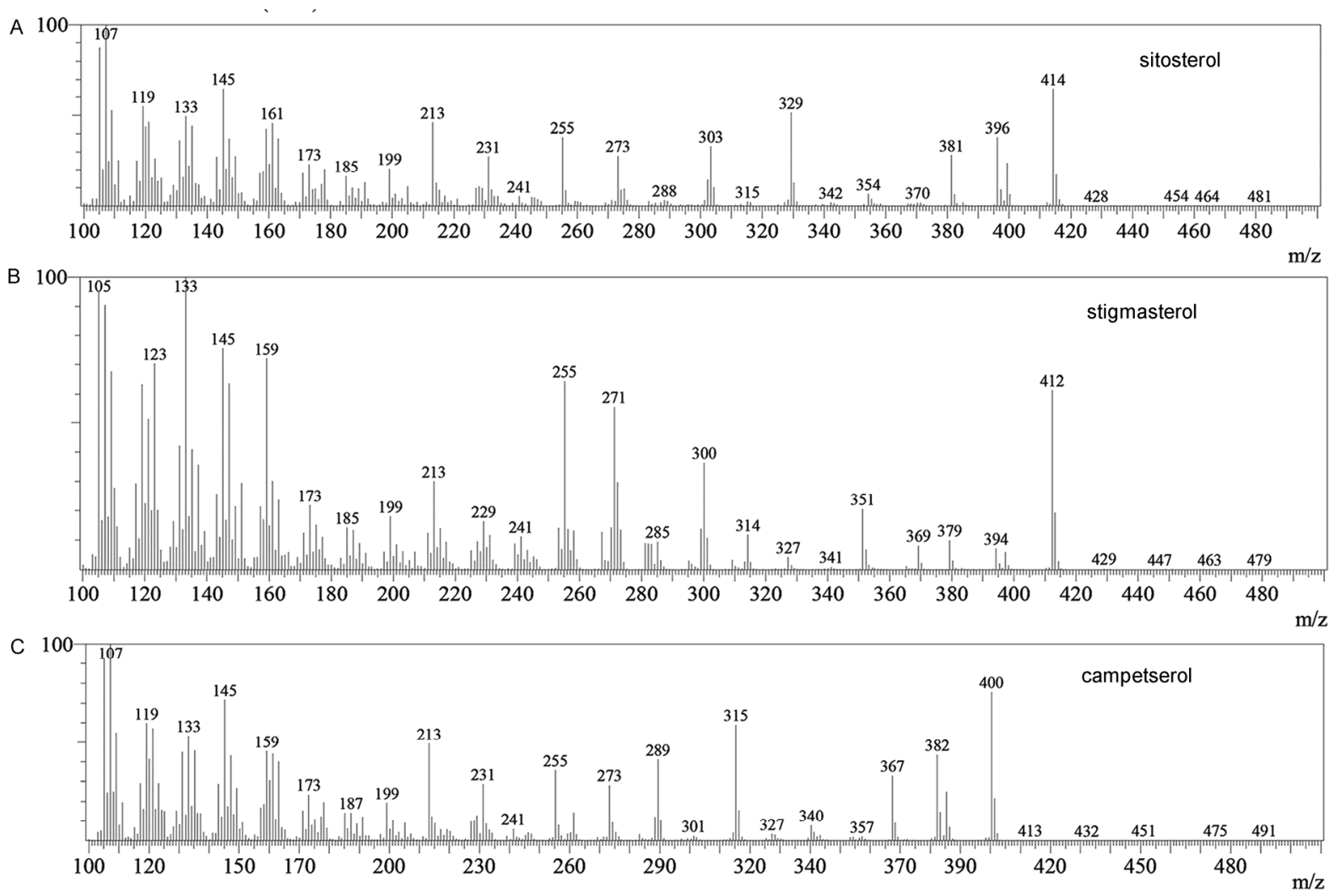

Figure 4 Mass ion fragmentation pattern of sitosterol, stigmasterol, and campesterol. A, MS pattern of sitosterol. The peak (414) is the molecular ion peak of sitosterol. B, MS pattern of stigmasterol. The peak of 412 is the molecular ion peak of stigmasterol. C, MS pattern of campesterol. The peak of 400 is the molecular ion peak of campesterol.

the wild-type fiber after 15 DPA. The speed of fiber elongation declined significantly with the decrease in sitosterol and campesterol concentrations between 15 and 20 DPA; the fiber ceased to elongate when the low levels of these phytosterols were maintained. The lower sitosterol and campesterol content in the $l i-l$ fiber compared to the 10-DPA fiber of wild type cotton suggested a lower elongation speed of the mutant, or that the elongation was terminated at 10 DPA. The decrease in phytosterol concentration suppresses membrane expansion and influences the activity of membrane binding proteins involved in cell elongation. On the other hand, a decrease in campesterol (precursor of BR biosynthesis) content might result in active BR deficiency, consequently suppressing fiber cell elongation. Phytosterols are implicated in a number of cell growth and development processes. The results of our study indicated that phytosterols play an important role in fiber cell elongation. However, the molecular mechanism of phytosterol action must be further analyzed.

Stigmasterol was not detected in the 6-15 DPA fibers, and detected in the 15-30 DPA fibers, indicating that stigmasterol may be associated with secondary cell wall synthesis. GhCYP710-1 and GhCYP710-2 (genes involved in the conversion of sitosterol to stigmasterol) expression was upregulated in the 6-DPA ovules (with fiber) and 10-DPA fibers of the $l i-1$ mutant. Stigmasterol was also detected in the 10-DPA mutant fiber; however, we observed no significant differences between the $l i-1$ mutant and TM- 1 wild type $(P>0.05)$. Secondary cell wall synthesis might have occurred in the 10-DPA fiber of $l i-1$ mutant. Consistent with this theory, the $l i-1$ fiber is a thick and short fiber cell, and stigmasterol is involved in the fruit maturation process (Bolton et al., 2009; Liu et al., 2012; Whitaker and Gapper, 2008). Therefore, the role of stigmasterols in secondary cell wall biosynthesis must be further characterized.

\section{Wave of phytosterol content and campesterol:sitosterol ratio in the fiber development process}

Cotton fiber cells undergo rapid elongation from 3 DPA and peak at approximately 10 DPA, following which the speed of elongation gradually decreases, with elongation being terminated at $\sim 20$ DPA. Secondary cell wall synthesis occurs between 15 and $20 \mathrm{DPA}$, and lasts up to $45 \mathrm{DPA}$ (Basra and Sukumar, 2000) (Figure 8). Corresponding to this, our results showed a rapid increase in sitosterol and campesterol concentrations from 6 DPA, with a peak at $\sim 10$ DPA; the concentrations decreased gradually, reaching the lowest level at 20 DPA. The concentrations of both sitosterol and campesterol were very low between 20 and 30 DPA (Figure 8). This indicated that high concentrations of 

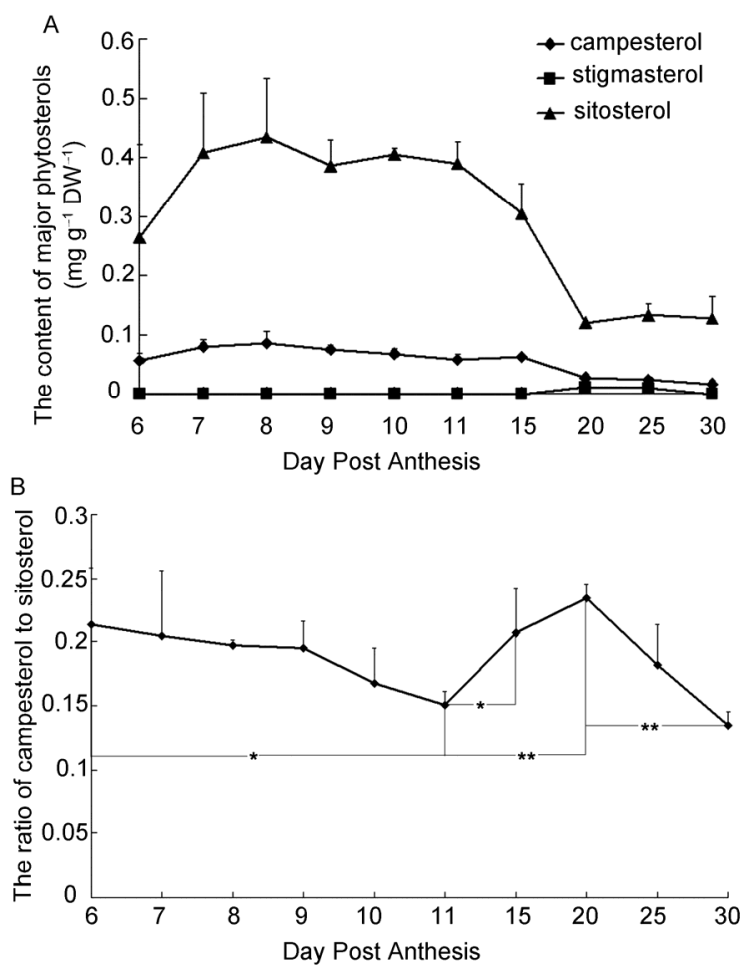

Figure 5 Time course of phytosterol production in the developing cotton fiber. A, Campesterol, sitosterol, and stigmasterol contents in the 6- to 30-DPA fibers. B, The ratio of campesterol to sitosterol in the 6- to 30-DPA fibers. Error bars indicate the SD for analyses of three replicate samples. The data was evaluated by Student's $t$-test to determine statistical significance. *: $P<0.05$; **: $P<0.01$.

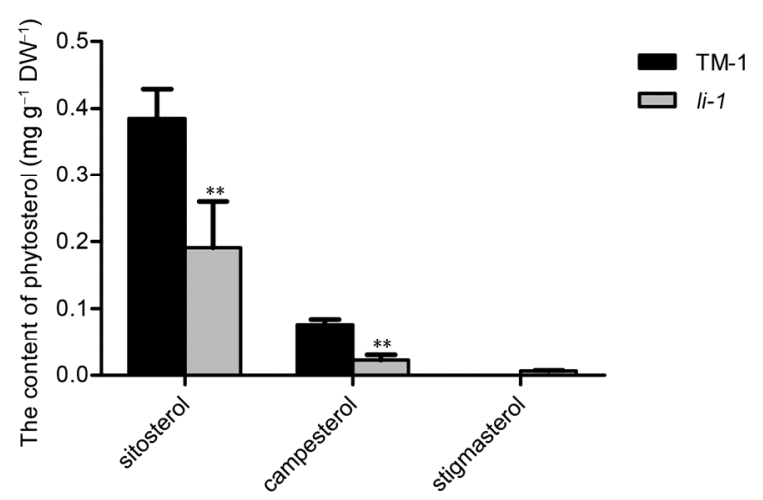

Figure 6 Phytosterol content in elongating fibers of $l i-1$ mutant and TM-1. Sitosterol, campesterol, and stigmasterol were detected in 10-DPA fibers of $l i-1$ mutant and TM-1 wild type. Error bars indicate SD for analyses of three replicate samples. The data was evaluated by Student's $t$-test to determine statistical significance. ${ }^{* *}: P<0.01$.

sitosterol and campesterol benefit fiber elongation. It is reasonable to assume that fiber cell elongation requires an increase in membrane constructs and cell elongation signal. Sitosterol is a membrane component, while campesterol is a precursor of BR, a signaling molecule related to plant cell elongation.

With respect to the speed of fiber elongation, the process can be divided into two stages: the early elongation stage (3-10 DPA) and the late elongation stage ( 10-20 DPA) (Meinert and Delmer, 1977). The rate of fiber elongation is accelerated during the early elongation stage, with the maximal rate occurring at $\sim 12 \mathrm{DPA}$, while the rate declines gradually during the late elongation stage (Meinert and Delmer, 1977; Schubert et al., 1973). Our data revealed a consistent decrease in the ratio of campesterol to sitosterol from 6 to 11 DPA, indicating a negatively correlation between this ratio and the rate of fiber elongation. Likewise, the ratio increased gradually from 11 to 20 DPA, indicating the negative correlation between the ratio and the rate of fiber elongation during the late elongation stage (Figures $5 \mathrm{~B}$ and 8). Therefore, a low ratio of campesterol to sitosterol was conducive for fiber elongation, while a high ratio suppressed fiber elongation, during the elongation stage (Figure 8). The relationship between the rate of elongation and the ratio indicated that campesterol and sitosterol were tightly regulated by fiber development, and that the coordination between the synthesis of membrane components (such as sitosterol) and that of signal molecule was essential for fiber elongation. The fiber structure may also be attributed to phytosterol activity during fiber growth. Previous reports have also revealed that phytosterols modulate the activity and distribution of membrane-bound proteins, such as receptors, enzymes, and components of the signaling pathway, in addition to regulating the membrane fluidity and permeability (Boutté and Grebe, 2009). A number of physiological and biochemical processes are activated during the elongation of fiber cells. However, the role of phytosterols in these processes remains to be elucidated.

\section{MATERIALS AND METHODS}

\section{Plant material and growth conditions}

Gossypium hirsutum ligon lintless-1 (li-1) mutant and Xuzhou 142 (Gossypium hirsutum cv. Xuzhou 142) were provided by the Cotton Research Institute, Chinese Academy of Agricultural Sciences, and were grown in a field with normal administration. TM-1, the mutant's isogenic wild-type line was among the progeny of the $l i-1$ mutant. Flowers were tagged on the day of anthesis.

\section{Isolation of total RNA and synthesis of first-strand cDNA}

The total RNA was isolated from 0-DPA ovules (with fibers), 6-DPA ovules (with fibers), 10-DPA fibers (without ovules), and 10-DPA ovules (without fibers) of the $l i-1 \mathrm{mu}-$ tant and the wild-type TM-1 using a standard kit (Aidlab, Beijing, China). Five micrograms of total RNA from each sample was used to synthesize the first-strand cDNA using the reverse transcriptase (RT) reagent kit containing a gDNA Eraser (TaKaRa, Dalian, China) according to the manufacturer's instructions. 

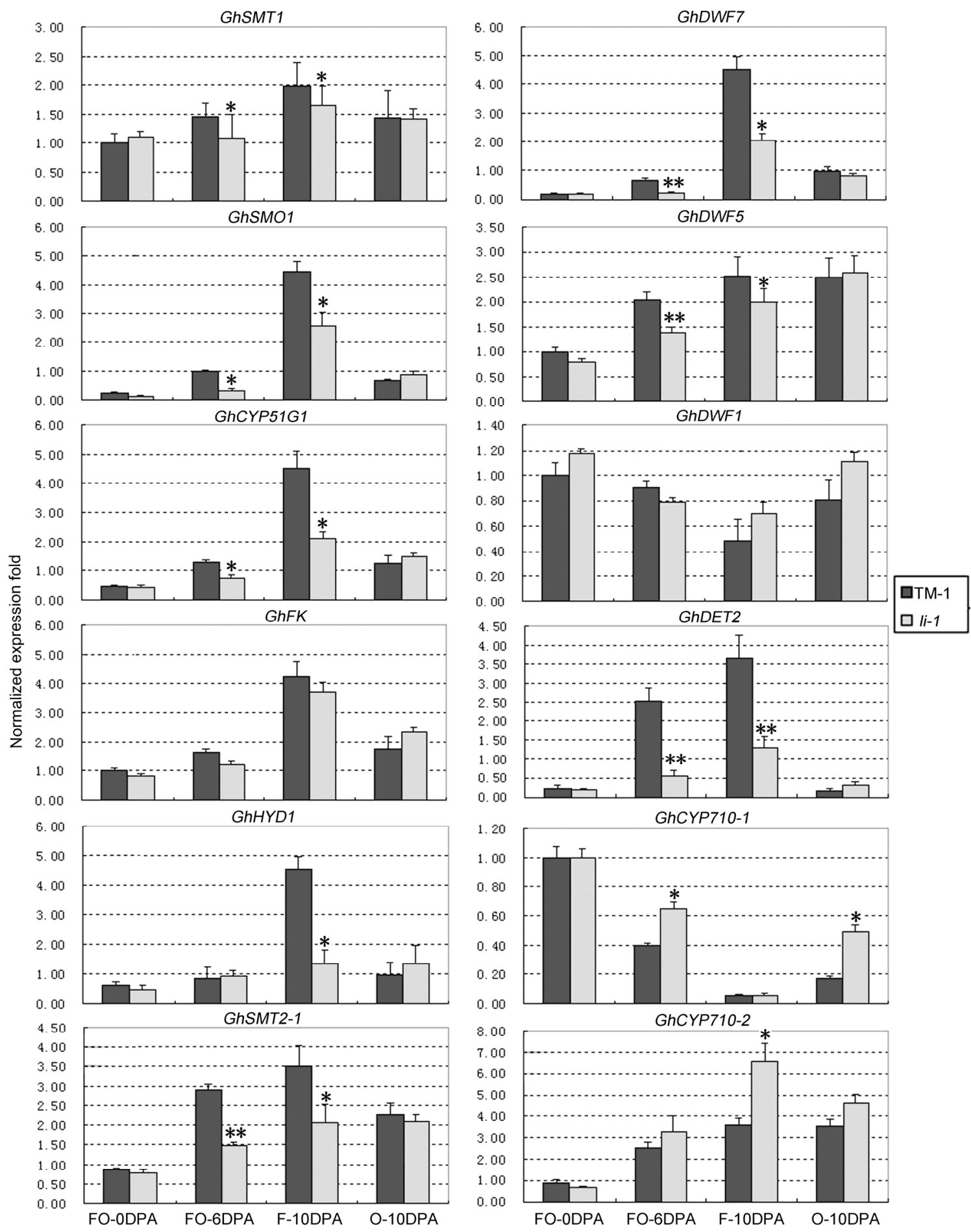

Figure 7 Comparison of transcripts of genes involved in phytosterol biosynthesis between the $l i-1$ mutant and TM-1. Total RNA was isolated from the following wild type (TM-1) and mutant (li-1) ovules and/or fiber cells: 0-DPA ovules (with fibers), 6-DPA ovules (with fibers), 10-DPA fibers (without ovules), and 10-DPA ovules (without fibers). The first strand cDNA (from total RNA) was used as the template for quantitative real-time reverse transcriptase polymerase chain reaction (RT-PCR), using cotton HISTONE3 for normalization of the data. FO-0DPA: 0-DPA ovules (with fibers); FO-6DPA: 6-DPA ovules (with fibers); F-10DPA: 10-DPA fibers (without ovules); O-10DPA: 10-DPA ovules (without fibers). Error bars represent the SD for three independent experiments. The data was evaluated by Student's $t$-test to determine statistical significance. $*: P<0.05$; **: $P<0.01$.

\section{Quantitative real-time RT-PCR and statistical analysis}

The synthesized first-strand cDNA was used as the template in quantitative real-time RT-PCR. The HISTONE3 gene from cotton was used to normalize the data. The primers for each gene are listed in Supplementary Table S1. Error bars 


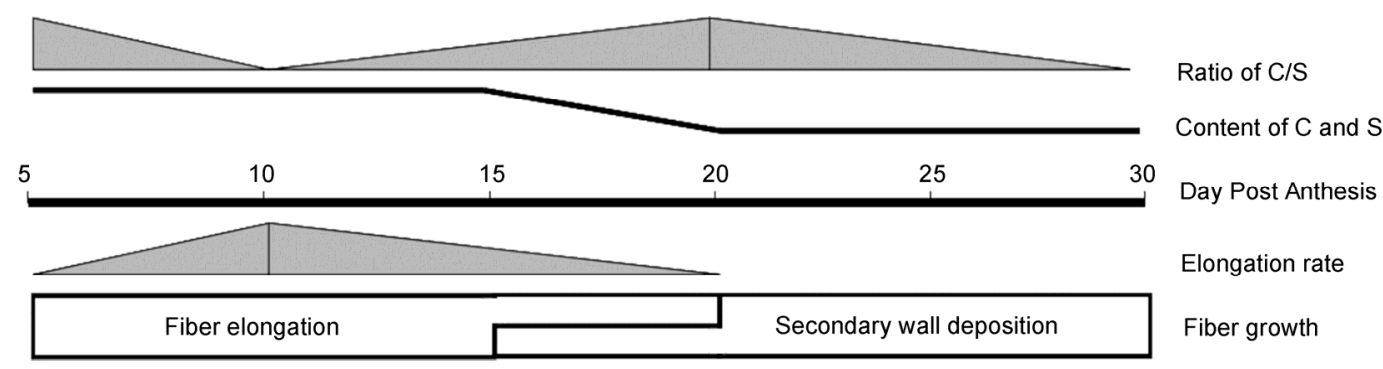

Figure 8 Relationship between fiber development, phytosterol content, and the ratio of campesterol to sitosterol. S: sitosterol; C: campesterol.

represent the standard deviation (SD) for three independent experiments. The data was evaluated by Student's $t$-test to obtain statistically significant values. Phytosterol biosynthetic genes are indicated in Supplementary Figure S1.

\section{In vitro ovule culture, fiber length measurement, and tridemorph treatment}

In vitro cotton ovule culture and fiber length measurement were conducted as described previously by Luo et al. (Luo et al., 2007). The sterol biosynthesis inhibitor, tridemorph (2,6-dimethyl-N-tridecyl-morpholine; Sigma-Aldrich, St. Louis, MO) was dissolved in $100 \%$ methanol to prepare a $1.25 \mathrm{mg} \mathrm{mL}^{-1}$ stock solution. Tridemorph was added to BT medium (Beasley and Ting, 1973, 1974) at final concentrations of $0.63,1.25$, and $2.50 \mathrm{mg} \mathrm{L}^{-1}$, respectively. BT medium adjusted with the amount of methanol equivalent to that used to dissolve tridemorph was used as the control.

\section{Tridemorph treatment in plants}

Four groups of plants ( 3 plants each) were randomly selected from the field. Tridemorph was exogenously applied according to the method detailed by Seagull and Giavalis (Seagull and Giavalis, 2004). One hundred microliters of 25 $\mathrm{mg} \mathrm{L}^{-1}$ tridemorph was applied to each developing boll at five day intervals from the day of anthesis (0 DPA) to 30 DPA. A large number of treated bolls were abscised prior to the harvest, and bolls treated with a mock were retained. The fibers were tested when the retained bolls reached maturity and opened. Deionized water, adjusted with the amount of methanol equivalent to that used to dissolve the tridemorph, was used for the mock treatment.

\section{Extraction of phytosterols}

The three major phytosterols were extracted and analyzed according to a previously described method (He et al., 2003). Briefly, the flowers developing on mutant and/or wild type cotton plants were marked on the day of anthesis. Cotton bolls were harvested, and fibers derived from the ovule were rapidly dried at $70^{\circ} \mathrm{C}$. The dried material was ground to powder and quantified. The sample was extracted with $50 \mathrm{~mL} \mathrm{MeOH} / \mathrm{CHCl}_{3}(5 / 1, \mathrm{v} / \mathrm{v}) ;{ }^{13} \mathrm{C}$-cholesterol was added as an internal standard. The sample was extracted at $70^{\circ} \mathrm{C}$ for $24 \mathrm{~h}$. The dried residue was saponified with $1 \mathrm{~mol} \mathrm{~L}^{-1}$ $\mathrm{NaOH}$ in methanol at $90^{\circ} \mathrm{C}$ for $2 \mathrm{~h}$ to release the sterol moi- ety of steryl esters. The saponified solution was evaporated at $90^{\circ} \mathrm{C}$ and dissolved in $200 \mathrm{~mL}$ water. The extract was partitioned three times between $\mathrm{CHCl}_{3}$ and water. The $\mathrm{CHCl}_{3}$-soluble fraction was evaporated, and the residues were dissolved in $5 \mathrm{~mL} \mathrm{CHCl}$ and transferred to the tube. The extract was dried and dissolved in $\mathrm{MeOH}$.

\section{Gas chromatography-Mass spectrometry (GC-MS) analysis}

GC-MS analysis was performed using a gas chromatograph mass spectrometer (GCMS-QP2010S; Shimadzu, Kyoto, Japan) under the following conditions: electron ionization, $70 \mathrm{eV}$; source temperature, $200^{\circ} \mathrm{C}$; injection temperature, $280^{\circ} \mathrm{C}$; column temperature program: $200^{\circ} \mathrm{C}$ for $1 \mathrm{~min}$, increased to $280^{\circ} \mathrm{C}$ at a rate of $10^{\circ} \mathrm{C} \mathrm{min}^{-1}$, and maintained at this temperature for $15 \mathrm{~min}$; interface temperature, $250^{\circ} \mathrm{C}$; carrier gas, He, flow rate, $1 \mathrm{~mL} \min ^{-1}$; and split injection ratio, $8: 1$.

Compliance and ethics The author(s) declare that they have no conflict of interest.

Acknowledgements This work was supported by the National Natural Science Foundation of China (31130039, 30671258), the Genetically Modified Organisms Breeding Major Projects, China (2009ZX08009-118B), and the Program for New Century Excellent Talents in University from the Ministry of Education, China (NCET-07-0712).

Asami, T., Nakano, T., and Fujioka, S. (2005). Plant Brassinosteroid Hormones. In: Gerald, L., ed. Vitamins \& Hormones. New York: Academic Press 479-504.

Basra, A.S., and Sukumar, S. (2000). Growth regulation of cotton fibers. In: Basra, A.S., ed. Cotton Fibers: Developmental Biology, Quality Improvement, and Textile Processing. New York: Food Products Press 47-58.

Basra, A.S., and Malik, C.P. (1984). Development of the cotton fiber. Int Rev Cytol 89, 65-113.

Beasley, C.A., and Ting, I.P. (1973). The effects of plant growth substances on in vitro fiber development from fertilized cotton ovules. Am J Bot 60, 130-139.

Beasley, C.A., and Ting, I.P. (1974). The effects of plant growth substances on in vitro fiber development from unfertilized cotton ovules. Am J Bot 61, 188-194.

Benveniste, P. (2004). Biosynthesis and accumulation of sterols. Annu Rev Plant Biol 55, 429-457.

Bolton, J.J., Soliman, K.M., Wilkins, T.A., and Jenkins, J.N. (2009). Aberrant expression of critical genes during secondary cell wall biogenesis 
in a cotton mutant, ligon lintless-1 (li-1). Comp Funct Genomics 2009, 659-601.

Boutté, Y., and Grebe, M. (2009). Cellular processes relying on sterol function in plants. Curr Opin Plant Biol 12, 705-713.

Cao, X. (2015). Whole genome sequencing of cotton-a new chapter in cotton genomics. Sci China Life Sci 58, 515-516.

Carland, F.M., Fujioka, S., Takatsuto, S., Yoshida, S., and Nelson, T. (2002). The identification of CVP1 reveals a role for sterols in vascular patterning. Plant Cell 14, 2045-2058.

Choe, S., Tanaka, A., Noguchi, T., Fujioka, S., Takatsuto, S., Ross, A.S., Tax, F.E., Yoshida, S., and Feldmann, K.A. (2000). Lesions in the sterol Delta (7) reductase gene of Arabidopsis cause dwarfism due to a block in brassinosteroid biosynthesis. Plant J 21, 431-443.

Chu, Z., Li, L., Song, L., and Xue, H. (2006). Advances on brassinosteroid biosynthesis and functions. Chin Bull Botany 23, 543-555

Claude, C., Alain, R., Maryse, T., and Yves, S. (1996). Effect of tridemorph and fenpropimorph on sterol composition in fenugreek. Phytochemistry 41, 423-431.

Clouse, S.D. (2000). Plant development: a role for sterols in embryogenesis. Curr Biol 10, R601-R604.

Clouse, S.D. (2002). Arabidopsis mutants reveal multiple roles for sterols in plant development. Plant Cell 14, 1995-2000.

Deng, F., Tu, L., Tan, J., Li, Y., Nie, Y., and Zhang, X. (2012). GbPDF1 is involved in cotton fiber initiation via the core cis-element HDZIP2ATATHB2. Plant Physiol 158, 890-904.

Fridman, Y., and Savaldi-Goldstein, S. (2013). Brassinosteroids in growth control: how, when and where. Plant Sci 209, 24-31.

Fujioka, S., and Yokota, T. (2003). Biosynthesis and metabolism of brassinosteroids. Annu Rev Plant Biol 54, 137-164.

Gou, J., Wang, L., Chen, S., Hu, W., and Chen, X. (2007). Gene expression and metabolite profiles of cotton fiber during cell elongation and secondary cell wall synthesis. Cell Res 17, 422-434.

Gudesblat, G.E., and Russinova, E. (2011). Plants grow on brassinosteroids. Curr Opin Plant Biol 14, 530-537.

He, J.X., Fujioka, S., Li, T.C., Kang, S.G., Seto, H., Takatsuto, S., Yoshida, S., and Jang, J.C. (2003). Sterols regulate development and gene expression in Arabidopsis. Plant Physiol 131, 1258-1269.

Kasukabe, Y., Fujisawa, Y., Nishiguchi, K., Maekawa, S., Allen, Y., and Dale, R. (2001) Production of cotton fiber with improved fiber characteristics. United States Patent, 20010018773.

Kim, H.B., Schaller, H., Goh, C.H., Kwon, M., Choe, S., An, C.S., Durst, F., Feldmann, K.A., and Feyereisen, R. (2005). Arabidopsis cyp51 mutant shows postembryonic seedling lethality associated with lack of membrane integrity. Plant Physiol 138, 2033-2047.

Lee, J.J., Woodward, A.W., and Chen, Z.J. (2007). Gene expression changes and early events in cotton fibre development. Ann Bot 100, 1391-1401.

Li, F., Fan, G., Lu, C., Xiao, G., Zou, C., Kohel, R.J., Ma, Z., Shang, H., Ma, X., Wu, J., Liang, X., Huang, G., Percy, R.G., Liu, K., Yang, W., Chen, W., Du, X., Shi, C., Yuan, Y., Ye, W., Liu, X., Zhang, X., Liu, W., Wei, H., Wei, S., Huang, G., Zhang, X., Zhu, S., Zhang, H., Sun, F., Wang, X., Liang, J., Wang, J., He, Q., Huang, L., Wang, J., Cui, J., Song, G., Wang, K., Xu, X., Yu, J., Zhu, Y., and Yu, S. (2015). Genome sequence of cultivated Upland cotton (Gossypium hirsutum TM-1) provides insights into genome evolution. Nat Biotechol 33, 524-530.

Liu, K., Sun, J., Yao, L., and Yuan, Y. (2012). Transcriptome analysis reveals critical genes and key pathways for early cotton fiber elongation in ligon lintless-1 mutant. Genomics 100, 42-50.

Luo, M., Tan, K., Xiao, Z., Hu, M., Liao, P., and Chen, K. (2008). Cloning and expression of two sterol C-24 methyltransferase genes from upland cotton (Gossypium hirsuturm L.). J Genet Genom 35, 357-363.

Luo, M., Xiao, Y., Li, X., Lu, X., Deng, W., Li, D., Hou, L., Hu, M., Li, Y., and Pei, Y. (2007). GhDET2, a steroid 5 $\alpha$-reductase, plays an important role in cotton fiber cell initiation and elongation. Plant $\mathrm{J} 51$, 419-430.

Meinert, M.C., and Delmer, D.P. (1977). Changes in biochemical composition of the cell wall of the cotton fiber during development. Plant
Physiol 59, 1088-1097.

Men, S., Boutte, Y., Ikeda, Y., Li, X., Palme, K., Stierhof, Y.D., Hartmann, M.A., Moritz,T., and Grebe, M. (2008). Sterol-dependent endocytosis mediates postcytokinetic acquisition of PIN2 auxin efflux carrier polarity. Nat Cell Biol 10, 237-244.

Neelakandan, A.K., Nguyen, H.T.M., Kumar, R., Tran, L.S.P., Guttikonda, S.K., Quach, T.N., Aldrich, D.L., Nes, W.D., and Nguyen, H.T. (2010). Molecular characterization and functional analysis of Glycine max sterol methyl transferase 2 genes involved in plant membrane sterol biosynthesis. Plant Mol Biol 74, 503-518.

Ovečka, M., Berson, T., Beck, M., Derksen, J., Šamaj, J., Baluška, F., and Lichtscheidl, I.K. (2010). Structural sterols are involved in both the initiation and tip growth of root hairs in Arabidopsis thaliana. Plant Cell 22, 2999-3019.

Padmalatha, K.V., Patil, D.P., Kumar, K., Dhandapani, G., Kanakachari, M., Phanindra, M.L.V., Kumar, S., Mohan, T.C., Jain, N., Prakash, A.H., Vamadevaiah, H., Katageri, I.S., Leelavathi, S., Reddy, M.K., Kumar, P.A., and Reddy, V.S. (2012). Functional genomics of fuzzless-lintless mutant of Gossypium hirsutum L. cv. MCU5 reveal key genes and pathways involved in cotton fibre initiation and elongation. BMC Genomics 13, 624.

Pei, Y. (2015). The homeodomain-containing transcription factor, GhHOX3, is a key regulator of cotton fiber elongation. Sci China Life Sci 58, 309-310.

Ryser, U. (2000). Cotton fiber initiation and histodifferentiation. In: Basra, A.S., ed. Cotton Fibers: Developmental Biology, Quality Improvement, and Textile Processing. New York: Food Products Press 1-34.

Schaeffer, A., Bronner, R., Benveniste, P., and Schaller, H. (2001). The ratio of campesterol to sitosterol which modulates growth in Arabidopsis is controlled by STEROL METHYLTRANSFERASE 2-1, Plant J $25,605-615$.

Schaller, H. (2003). The role of sterols in plant growth and development. Prog Lipid Res 42, 163-175.

Schaller, H. (2004). New aspects of sterol biosynthesis in growth and development of higher plants. Plant Physiol Biochem 42, 465-476.

Schrick, K., Fujioka, S., Takatsuto, S., Stierhof, Y.D., Stransky, H., Yoshida, S., and Jürgens, G. (2004). A link between sterol biosynthesis, the cell wall, and cellulose in Arabidopsis. Plant J 38, 227-243.

Schrick, K., Mayer, U., Horrichs, A., Kuhnt, C., Bellini, C., Dangl, J., Schmidt, J., and Jürgens, G. (2000). FACKEL is a sterol C-14 reductase required for organized cell division and expansion in Arabidopsis embryogenesis. Genes Dev 14,1471-1484.

Schrick, K., Mayer, U., Martin, G., Bellini, C., Kuhnt, C., Schmidt, J., and Jürgens, G. (2002). Interactions between sterol biosynthesis genes in embryonic development of Arabidopsis. Plant J 31, 61-73.

Schubert, A.M., Benedict, C.R., Berlin, J.D., and Kohel, R.J. (1973). Cotton fiber development-kinetics of cell elongation and secondary wall thickening. Crop Sci 13, 704-709.

Seagull, R.W., and Giavalis, S. (2004). Pre- and post-anthesis application of exogenous hormones alters fiber production in Gossypium hirsutum L., cultivar Maxxa GTO. J Cotton Sci 8, 105-111.

Shan, C., Shangguan, X., Zhao, B., Zhang, X., Chao, L., Yang, C., Wang, L., Zhu, H., Zeng, Y., Guo, W., Zhou, B., Hu, G., Guan, X., Chen, Z., Wendel, J., Zhang, T., and Chen, X. (2014). Control of cotton fibre elongation by a homeodomain transcription factor GhHOX3. Nat Commun 5, 5519.

Shi, Y., Zhu, S., Mao, X., Feng, J., Qin, Y., Zhang, L., Cheng, J., Wei, L., Wang, Z., and Zhu, Y. (2006). Transcriptome profiling, molecular biological, and physiological studies reveal a major role for ethylene in cotton fiber cell elongation. Plant Cell 18, 651-664.

Souter, M., Topping, J., Pullen, M., Friml, J., Palme, K., Hackett, R., Grierson, D., and Lindsey, K. (2002). hydra mutants of Arabidopsis are defective in sterol profiles and auxin and ethylene signaling. Plant Cell 14, 1017-1031.

Souter, M.A., Pullen, M.L., Topping, J.F., Zhang, X., and Lindsey, K. (2004). Rescue of defective auxin-mediated gene expression and root meristem function by inhibition of ethylene signalling in sterol biosynthesis mutants of Arabidopsis. Planta 219, 773-783.

Sun, Y., and Allen, R.D. (2005). Functional analysis of the BIN2 genes of 
cotton. Mol Genet Genomics 274, 51-59.

Sun, Y., Fokar, M., Asami, T., Yoshida, S., and Allen, R.D. (2004). Characterization of the brassinosteroid insensitive 1 genes of cotton. Plant Mol Biol 54, 221-232.

Sun, Y., Veerabomma, S., Abdel-Mageed, H.A., Fokar, M., Asami, T., Yoshida, S., and Allen, R.D. (2005). Brassinosteroid regulates fiber development on cultured cotton ovules. Plant Cell Physiol 46, 1384-1391.

Suzuki, M., Nakagawa, S., Kamide, Y., Kobayashi, K., Ohyama, K., Hashinokuchi, H., Kiuchi, R., Saito, K., Muranaka, T., and Nagata, N. (2009). Complete blockage of the mevalonate pathway results in male gametophyte lethality. J Exp Bot 60, 2055-2064.

Tan, K., Hu, M., Li, X., Qin, S., Li, D., Luo, X., Zhao, J., Zang, Z., Li, B., Pei, Y., and Luo, M. (2009). Molecular identification and expression analysis of GhCYP51G1 gene, a homologue of obtusifoliol-14alpha-demethylase gene, from upland cotton. Acta Agronom Sin 35, 1194-1201.

Topping, J.F., May, V.J., Muskett, P.R., and Lindsey, K. (1997). Mutations in the HYDRA1 gene of Arabidopsis perturb cell shape and disrupt embryonic and seedling morphogenesis. Development 124, 4415-4424.

Wanjiea, S.W., Weltib, R., Moreauc, R.A., and Chapman, K.D. (2005). Identification and quantification of glycerolipids in cotton fibers: reconciliation with metabolic pathway predictions from DNA databases. Lipids 40, 773-785.

Whitaker, B.D., and Gapper, N.E. (2008). Ripening-specific stigmasterol increase in tomato fruit is associated with increased sterol C-22 desaturase (CYP710A11) gene expression. J Agric Food Chem 56, 38283835 .

Willemsen, V., Friml, J., Grebe, M., Toorn, A.V.D., Palme, K., and Scheres, B. (2003). Cell polarity and PIN protein positioning in Arabidopsis require STEROL METHYLTRANSFERASE1 function. Plant Cell 15, 612-625.

Williams, M.E. (2011). Brassinosteroids: plant steroid hormones. Teaching Tools in Plant Biology: Lecture Notes. Plant Cell doi/10.1105/tpc. 110.tt0910

Xiao, Y., Li, D., Yin, M., Li, X., Zhang, M., Wang, Y., Dong, J., Zhao, J., Luo, M., Luo, X., Hou, L., Lin, H., and Pei, Y. (2010). Gibberellin 20-oxidase promotes initiation and elongation of cotton fibers by regulating gibberellin synthesis. J Plant Physiol 167, 829-837.

Yang, S., Cheung, F., Lee, J.J., Ha, M., Wei, N., Sze, S.H., Stelly, D.M., Thaxton, P., Triplett, B., Town, C.D., and Chen, Z. (2006). Accumulation of genome-specific transcripts, transcription factors and phytohormonal regulators during early stages of fiber cell development in allotetraploid cotton. Plant J 47, 761-775.

Yang, Z., Zhang, C., Yang, X., Liu, K., Wu, Z., Zhang, X., Zheng, W., Xun, Q., Liu, C., Lu, L., Yang, Z., Qian, Y., Xu, Z., Li, C., Li, J., and Li, F. (2014). PAG1, a cotton brassinosteroid catabolism gene, modulates fiber elongation. New Phytol 203, 437-448.

Zang, Z., Hu, M., Li, X., Chen, K., Liao, P., Xiao, Y., Hou, L., Pei, Y., Luo, M. (2011). Molecular identification and expression analysis of GhHYDRAl gene, a homologue of HYDRA1 gene from upland cotton (Gossypium hirsutum L.). Agri Sci China 10, 41-48.

Zhang, M., Zheng, X., Song, S., Zeng, Q., Hou, L., Li, D., Zhao, J., Wei, Y., Li, X., Luo, M., Xiao, Y., Luo, X., Zhang, J., Xiang, C., and Pei, Y. (2011). Spatiotemporal manipulation of auxin biosynthesis in cotton ovule epidermal cells enhances fiber yield and quality. Nat Biotechnol 29, 453-458.

Zhang, T., Hu, Y., Jiang, W., Fang, L., Guan, X., Chen, J., Zhang, J., Saski, C.A., Scheffler, B.E., Stelly, D.M., Hulse-Kemp, A.M., Wan, Q., Liu, B., Liu, C.X., Wang, S., Pan, M.Q., Wang, Y.K., Wang, D., Ye, W., Chang, L., Zhang, W., Song, Q., Kirkbride, R.C., Chen, X., Dennis, E., Llewellyn, D.J., Peterson, D.G., Thaxton, P., Jones, D.C., Wang, Q., Xu, X., Zhang, H., Wu, H., Zhou, L., Mei, G., Chen, S., Tian, Y., Xiang, D., Li, X., Ding, J., Zuo, Q., Tao, L., Liu, Y., Li, J., Lin, Y., Hui, Y., Cao, Z., Cai, C., Zhu, X., Jiang, Z., Zhou, B., Guo, W., Li, R., and Chen, Z. (2015). Sequencing of allotetraploid cotton (Gossypium hirsutum L. acc. TM-1) provides a resource for fiber improvement. Nat Biotechol 33, 531-537.

Open Access This article is distributed under the terms of the Creative Commons Attribution License which permits any use, distribution, and reproduction in any medium, provided the original author(s) and source are credited.

\section{SUPPORTING INFORMATION}

Table S1 Primers used for quantitative real-time RT-PCR.

Figure S1 Model for the phytosterol biosynthesis pathway in Arabidopsis thaliana.

The supporting information is available online at life.scichina.com and link.springer.com. The supporting materials are published as submitted, without typesetting or editing. The responsibility for scientific accuracy and content remains entirely with the authors. 